\title{
High Thermo-Optic Coefficient of Silicon Oxycarbide Photonic Waveguides
}

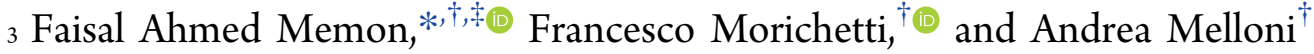 \\ $4{ }^{\dagger}$ Dipartimento di Elettronica, Informazione e Bioingegneria (DEIB), Politecnico di Milano, Via Ponzio 34/5, 20133 Milan, Italy \\ 5 Department of Telecommunications Engineering, Mehran University of Engineering \& Technology, Jamshoro 76062, Pakistan
}

6 ABSTRACT: In this Letter, we report on the observation of a high thermo-optic
7 coefficient (TOC) of silicon oxycarbide ( $\mathrm{SiOC}$ ) films deposited by reactive RF
8 magnetron sputtering for integrated photonic waveguides. In the $1550 \mathrm{~nm}$ wavelength
9 range, the measured TOC of SiOC is as large as $2.5 \times 10^{-4} \mathrm{RIU}{ }^{\circ} \mathrm{C}^{-1}$, which is about 30
10 times larger than that of silica and almost twice that of silicon. Thin films of SiOC have
11 been integrated in germanium-doped silica and silicon oxynitride conventional
12 waveguide technology, achieving a $10 \times$ and $3 \times$ enhancement of the waveguide
13 effective TOC, respectively. These results demonstrate the potential of SiOC for the
14 realization of highly efficient phase actuators and low-power-consumption thermally
15
tunable photonic integrated platforms.

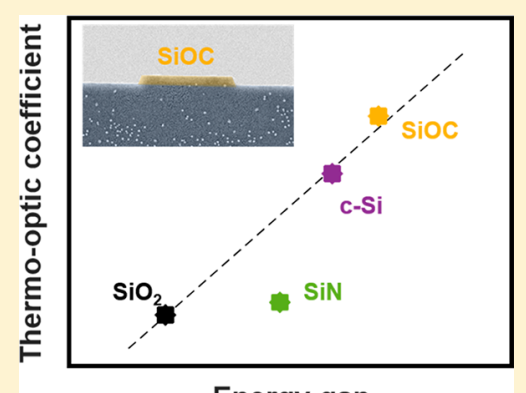

KEYWORDS: integrated photonics, thermo-optic effect, silicon oxycarbide, optical waveguides
17 mong the currently available integrated photonics plat18 forms, dielectric technologies are the most consolidated, 19 reliable, and widely exploited to realize commercial photonic 20 integrated circuits (PICs). Conventional dielectric waveguide 21 technologies include germanium-doped silica glass $\left(\mathrm{Ge}: \mathrm{SiO}_{2}\right)$, 22 silicon oxynitride $(\mathrm{SiON})$, and silicon nitride $\left(\mathrm{Si}_{3} \mathrm{~N}_{4}\right){ }^{1,2}$ These 23 technologies have long been used to fulfill the requirements of 24 photonic industries and enabled the fabrication of low-loss, 25 low-cost, polarization-independent, efficient fiber-coupled, and 26 relatively compact complex devices.

27 The tuning and reconfiguration of PICs is typically achieved 28 through heaters that induce a phase shift in the propagating 29 light by the thermo-optic effect. In dielectrics the thermo-optic 30 coefficient $d \mathrm{n} / d \mathrm{~T}$ (TOC) is quite low, on the order of $10^{-5}$ ${ }_{31}{ }^{\circ} \mathrm{C}^{-1}$. On one hand, it can be seen as an advantage because it 32 guarantees a weak dependence of PIC behavior on temperature 33 variations; on the other hand, the efficiency of the heaters is 34 rather low, as large electrical dissipation and large operational 35 temperatures are required for control operations. Devices 36 based on $\mathrm{SiO}_{2}, \mathrm{SiON}$, and $\mathrm{Si}_{3} \mathrm{~N}_{4}$ show a spectral shift of about $371 \mathrm{GHz} /{ }^{\circ} \mathrm{C}$, heaters are typically a few millimeters long, and the 38 electrical power dissipated to induce a $\pi$ phase shift is some 39 hundreds of milliwatts. The high control temperatures 40 introduce stringent requirements on the packaging and on 41 the need of power-hungry Peltier cells to sink the heat. Further, 42 the high temperatures on chip impact the reliability of the 43 heaters, which deteriorate with time.

44 In order to realize energy-efficient and reliable reconfig45 urable PICs, materials with a large TOC could be integrated in 46 conventional dielectric platforms. Examples of high-TOC 47 materials employed in optical waveguides include silicon (1.8 $\left.48 \times 10^{-4}{ }^{\circ} \mathrm{C}^{-1}\right)$ and ultrarich silicon nitride $\left(2.66 \times 10^{-4}{ }^{\circ} \mathrm{C}^{-1}\right){ }^{3}$ 49 High negative TOC values have been reported in polymers and $50 \mathrm{TiO}_{2}{ }^{4}$ which are mainly related to the thermal expansion.
Silicon oxycarbide (SiOC) is a novel class of glass 51 compounds that has gained momentum in the scientific 52 community and adopted in technologically important 53 applications such as interlayer dielectrics, anode material in 54 lithium-ion batteries, and photoluminescence. ${ }^{5-7}$ Recently, we 55 have introduced $\mathrm{SiOC}$ as a potential platform in integrated 56 photonics. ${ }^{8,9}$ The refractive index of SiOC can be tailored from 57 $n=1.45$, corresponding to $\mathrm{SiO}_{2}$, to about 3.2, for amorphous 58 SiC. ${ }^{10-12} \mathrm{SiOC}$ has the advantage of a low absorption 59 coefficient in the near-infrared region, and low-loss waveguides 60 on silica realized by sputtering have been demonstrated for the 61 first time with a core index of 1.578 and 2.2. ${ }^{8,9}$ Waveguide 62 attenuation of 2 to $4 \mathrm{~dB} / \mathrm{cm}$ in the telecom window of 155063 $\mathrm{nm}$ has been measured. In comparison with $\mathrm{SiON}$, exhibiting a 64 pronounced absorption peak around $1505 \mathrm{~nm}$ due to $\mathrm{N}-\mathrm{H} 65$ bonds, ${ }^{1}$ SiOC absorption is spectrally flat across the full (S, C, 66 L) telecom bands, offering a tremendous advantage for 67 broadband applications.

In this Letter, we report on silica-buried SiOC channel 69 waveguides where we observed a record TOC about 30 times 70 larger than that of silica-based waveguides and even higher 71 than that of silicon waveguides. Further, we propose and 72 demonstrate the integration of SiOC films with classical 73 dielectric waveguide technologies to enhance the efficiency of 74 thermo-optic phase actuators. Results suggest that the high 75 TOC of SiOC can be effectively exploited to achieve an 76 energy-efficient reconfiguration of integrated photonic circuits 77

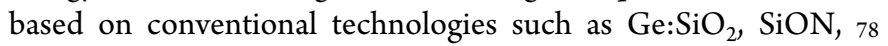
$\mathrm{SiN}$, and others.

Published: June 15, 2018 
80 The cross section of the silica-buried SiOC channel f1 81 waveguide is shown in Figure la together with a scanning

(a)

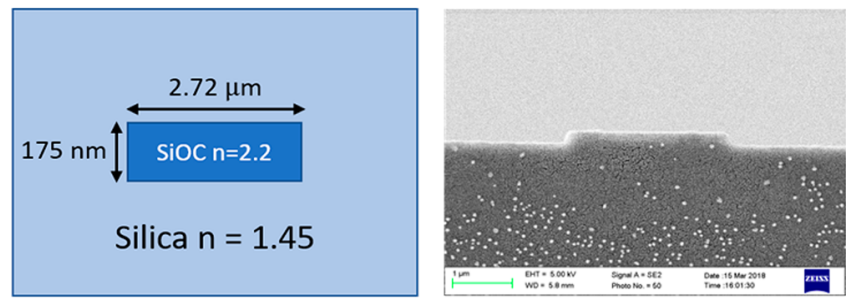

(b)

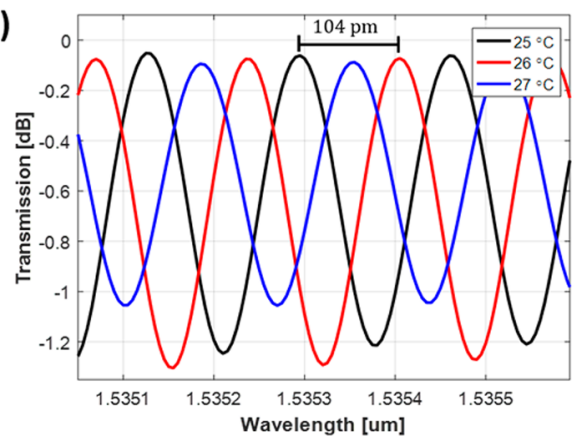

Figure 1. (a) Schematic and SEM photograph of the cross section of the SiOC channel waveguide. (b) Spectral response of a straight waveguide for three different temperatures.

82 electron microscopy (SEM) microphotograph of an uncovered 83 waveguide. The $\mathrm{SiOC}$ core of the waveguide has a rectangular 84 shape $\left(2.72 \times 0.175 \mu \mathrm{m}^{2}\right)$ and a refractive index of 2.2 . The $85 \mathrm{SiOC}$ film was deposited on a silicon wafer with a $6 \mu \mathrm{m}$ thick 86 silica substrate by using reactive radio frequency (RF) 87 magnetron sputtering from a $\mathrm{SiC}$ target in an oxygen and 88 argon atmosphere. Details on the SiOC sputtering procedure 89 can be found in previous publications. ${ }^{8,9}$ X-ray photoelectron 90 spectroscopy (XPS) measurements revealed that the deposited 91 material con $45 \%$ silicon, $27 \%$ oxygen, and $27 \%$ carbon, so 92 that the actual chemical composition is $\mathrm{Si}_{0.45} \mathrm{O}_{0.27} \mathrm{C}_{0.27}$ (for 93 brevity, in the remaining of the paper it will be simply referred 94 to as SiOC omitting the subscripts). Good adhesion of the $95 \mathrm{SiOC}$ film on the silica substrate was observed, with no 96 evidence of either pin holes or porosity. The SiOC surface 97 roughness, as estimated with atomic force microscopy (AFM), 98 is as low as $0.24 \mathrm{~nm} \mathrm{rms,} \mathrm{which} \mathrm{is} \mathrm{comparable} \mathrm{to} \mathrm{the} \mathrm{silica}$ 99 substrate. The optical constants of the SiOC film were 100 measured with a variable-angle spectroscopic ellipsometer over 101 a broad spectral range from the UV to near-infrared regions. ${ }^{9}$ 102 Around a wavelength of $1550 \mathrm{~nm}$ the measured refractive 103 index of the SiOC layer is about 2.2 and the extinction 104 coefficient is less than $10^{-4}$ above $600 \mathrm{~nm}$.

105 The waveguide cross section was defined by direct-laser106 writing lithography using an AZ 5214E photoresist and etching 107 with a reactive ion etching (RIE) machine. The RIE process of 108 mixed gases $\mathrm{CHF}_{3}(100 \mathrm{sccm})$ and $\mathrm{O}_{2}(5 \mathrm{sccm})$ was run 109 applying an RF power of $50 \mathrm{~W}$ and inductively coupled plasma 110 (ICP) power of $250 \mathrm{~W}$ for $20 \mathrm{~min}$. The etch rate was $9 \mathrm{~nm} /$ 111 min. After the SiOC core patterning, plasma enhanced 112 chemical vapor deposition (PECVD) silica with $n=1.45$ was 113 deposited as the upper cladding material. The strip-shaped 114 waveguide was selected in order to have good transverse 115 electric (TE) mode properties, with a small contribution of the 116 sidewall roughness to the waveguide attenuation. Several waveguides with different widths, ranging from 2 to $4 \mu \mathrm{m}$, were 117 fabricated and tested. The waveguide propagation losses 118 measured by cut-back technique are about $2 \mathrm{~dB} / \mathrm{cm}$. 119

The TOC of SiOC was estimated by optical transmission 120 measurement of the fabricated waveguides at different 121 temperatures. The photonic sample was mounted on a holder 122 whose temperature was controlled by a thermoelectric Peltier 123 module with a temperature accuracy within $0.1{ }^{\circ} \mathrm{C}$. Lensed 124 optical fibers were used to launch the light from a tunable laser, 125 and the polarization state of light was controlled with a 126 polarization controller guaranteeing at least $-30 \mathrm{~dB}$ crosstalk 127 between TE and transverse magnetic (TM) polarizations. TE- 128 polarized light was coupled to the SiOC waveguides from a 129 tunable laser operating in the broad spectral range from 1520130 to $1580 \mathrm{~nm}$.

Figure $1 \mathrm{~b}$ shows the measured transmission spectrum of a 132 SiOC waveguide around a wavelength $\lambda=1535.3 \mathrm{~nm}$ at three 133 different temperatures. The Fabry-Pérot fringes due to the 134 glass-air reflections at the waveguide input/output facets have 135 a period $\Delta \lambda=172 \mathrm{pm}$. The waveguide length is $L=3.5 \mathrm{~mm} 136$ and the effective group index results $n_{\mathrm{g}}=\lambda^{2} / 2 L \Delta \lambda=1.95 .137$ When the temperature is increased by $1{ }^{\circ} \mathrm{C}$, for instance from 138 $25{ }^{\circ} \mathrm{C}$ (black curve) to $26^{\circ} \mathrm{C}$ (red curve), a wavelength shift of 139 the waveguide transmission spectrum by $\mathrm{d} \lambda / \mathrm{d} T=104 \mathrm{pm} /{ }^{\circ} \mathrm{C} \quad 140$ is observed. The same wavelength shift was measured also 141 from 26 to $27{ }^{\circ} \mathrm{C}$ (blue curve) and for any additional 142 temperature degree. The effective thermo-optic coefficient of 143 the waveguide, hereinafter referred to as $K_{\text {eff }}$ is given by $\quad 144$

$$
K_{\text {eff }}=\frac{n_{g} \mathrm{~d} \lambda}{\lambda \mathrm{d} T}
$$

and results to be $K_{\text {eff }}=1.34 \times 10^{-4}{ }^{\circ} \mathrm{C}^{-1}$, a factor 10 larger 146 than that of $\mathrm{Si}_{3} \mathrm{~N}_{4}$ waveguides and 15 with respect to $\mathrm{SiO}_{2}-147$ based waveguides.

The thermo-optic coefficient of the SiOC material can be 149 evaluated by considering the overlap of the optical mode with 150 all the materials the waveguide is made of. With a variational 151 approach the perturbation of the phase propagation constant $\beta 152$ due to a variation $\Delta \varepsilon(x, y)$ of the dielectric constant of the 153 waveguide is given by ${ }^{13,14}$

$$
P \Delta \beta=\omega \iint_{A} \Delta \varepsilon(x, y) \mathbf{E E}^{*} \mathrm{~d} x \mathrm{~d} y
$$

where $\mathbf{E}$ is the electric field of the guided mode, $P$ is the power 156 carried by the mode, and the integral is over the entire 157 waveguide cross section $A$. Assuming that the perturbation is 158 induced by a change of temperature $\mathrm{d} T, \Delta \beta=\omega K_{\text {eff }} \mathrm{d} T / c$ and, 159 for each material, $\Delta \varepsilon=2 n K \mathrm{~d} T$, where $K$ is the material TOC. 160

The effective thermo-optic coefficient $K_{\text {eff }}$ can hence be 161 written as a combination of the contribution of the various 162 materials,

$$
K_{\text {eff }}=\sum_{i} K_{i} \Gamma_{i}
$$

where $K_{i}$ is the thermo-optic coefficient of the $i$ th material and 165

$$
\Gamma_{i}=\frac{c \iint_{A_{i}}\left[\varepsilon o \vec{E} \cdot \vec{E}^{*}\right] \mathrm{d} x \mathrm{~d} y}{\iint_{A}\left[\varepsilon o \vec{E}_{t} \times \vec{H}_{t}^{*}\right] \cdot \hat{z} \mathrm{~d} x \mathrm{~d} y}
$$

is the confinement factor of the mode in the considered 167 material, which is evaluated across a cross-sectional area $A_{\mathrm{i}} \cdot 168$ From eq 3 and the measured value of $K_{\text {eff }}$ of the waveguide, the 169 
170 thermo-optic coefficient of the $\mathrm{SiOC}$ material is $K_{\mathrm{SiOC}}=2.5 \times$ $17110^{-4}{ }^{\circ} \mathrm{C}^{-1}$, one of the largest values ever reported for dielectric 172 materials. Table 1 reports the values of the confinement factor

Table 1. Materials and Waveguide Parameters

\begin{tabular}{|c|c|c|c|}
\hline technology & SiOC wg & wg & ring \\
\hline group index $n_{\mathrm{g}}$ & 1.95 & 1.93 & 1.574 \\
\hline index contrast & $34 \%$ & $1.5 \%$ & $5.7 \%$ \\
\hline $\operatorname{size}\left[\mu \mathrm{m}^{2}\right]$ & $2.72 \times 0.175$ & $3.5 \times 2.8$ & $2.5 \times 1.8 \times 0.4$ \\
\hline$\Delta \lambda[\mathrm{pm}] /{ }^{\circ} \mathrm{C}$ & 104 & 130 & 31.3 \\
\hline$\Gamma_{\mathrm{SiO} 2}$ & 0.59 & 0.59 & 0.26 \\
\hline$\Gamma_{\mathrm{SiOC}}$ & 0.53 & 0.46 & 0.049 \\
\hline$\Gamma_{\mathrm{SiON}}$ & & & 0.72 \\
\hline $\begin{array}{c}K_{\text {eff }} \times 10^{-4} \\
(\text { expt })\end{array}$ & 1.34 & 1.62 & 0.32 \\
\hline
\end{tabular}

173 in the $\mathrm{SiOC}\left(\Gamma_{\mathrm{SiOC}}=0.53\right)$ and $\mathrm{SiO}_{2}\left(\Gamma_{\mathrm{SiO} 2}=0.589\right)$ layers of 174 the waveguide, as evaluated from electromagnetic simulations. 175 The thermo-optic coefficient of silica is $K_{\mathrm{SiO} 2}=0.9 \times 10^{-5}$ $176{ }^{\circ} \mathrm{C}^{-1}$.

177 The high TOC of SiOC, together with the transparency at 178 telecom wavelengths and the high refractive index, suggests its 179 use as a potential candidate for high integration scale and low 180 control power integrated optics. Here, we propose and 181 demonstrate the exploitation of $\mathrm{SiOC}$ for realizing highly 182 efficient thermo-optic phase shifters in classical and well183 consolidated photonic technologies, such as Ge-doped $\mathrm{SiO}_{2}$ 184 and $\mathrm{SiON}$ waveguides.

185 A thin layer of SiOC was deposited on the core of the optical 186 waveguides, which was then buried under a PECVD silica 187 upper cladding. The cross sections and micrographs of the $188 \mathrm{SiOC}$-coated $\mathrm{Ge}: \mathrm{SiO}_{2}$ waveguide and $\mathrm{SiON}$ ring resonator are 189 shown in Figure 2. The index contrast of the $\mathrm{Ge}: \mathrm{SiO}_{2}$ 190 waveguide is $1.5 \%$, and the core size is $3.5 \times 2.8 \mu \mathrm{m}^{2}$; for 191 the $\mathrm{SiON}$ waveguide, a rib shape with a size of $2.5 \times 1.8 \mu \mathrm{m}^{2}$ 192 (slab height of $0.4 \mu \mathrm{m}$ ) is used, the index contrast being equal 193 to $5.7 \%$. Waveguide data are summarized in Table 1 , and

(a)
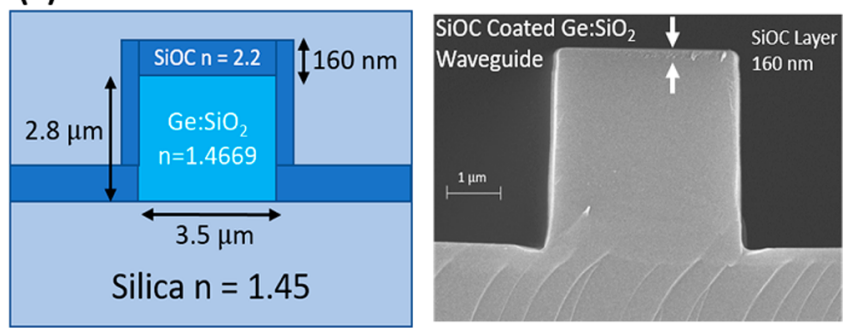

(b)
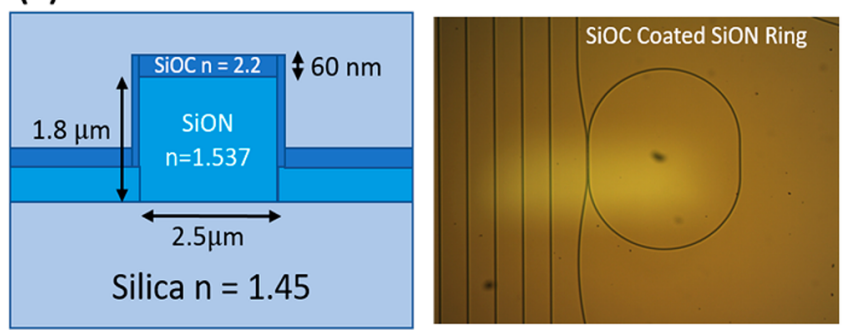

Figure 2. Cross section and microphotograph of (a) a $\mathrm{Ge}: \mathrm{SiO}_{2}$ waveguide coated with a SiOC layer; (b) a SiOC-coated $\mathrm{SiON}^{2}$ waveguide and ring resonator. details of their respective fabrication and optical character- 194 ization can be found in specific contributions. ${ }^{15,16}$ Electro- 195 magnetic simulations were performed to optimize the overlap 196 of the guided mode with the SiOC layer and limit the 197 perturbation on the waveguides. The thickness of the 198

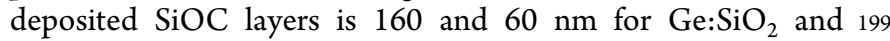
$\mathrm{SiON}$ waveguiding structures, respectively. The thickness of 200 the deposited SiOC films on the waveguide sidewalls is about 201 $40 \%$ of the top SiOC layer thickness, as estimated from the 202 SEM image given in Figure 2a.

Experiments were performed on $\mathrm{Ge}: \mathrm{SiO}_{2}$ straight wave- 204 guides and $\mathrm{SiON}$ ring resonators coated with the SiOC film. 205 The radius of the ring resonator is $555.3 \mu \mathrm{m}$, the coupler 206 length is $260 \mu \mathrm{m}$, and the physical length of the cavity is 4.009207 $\mathrm{mm}$. As in the case of the SiOC-core waveguide discussed in 208 Figure $1, K_{\text {eff }}$ was estimated by optical transmission measure- 209 ments of the fabricated structures at different temperatures. 210 Figure $3 \mathrm{a}$ shows the measured optical transmission spectrum of $211 \mathrm{f} 3$

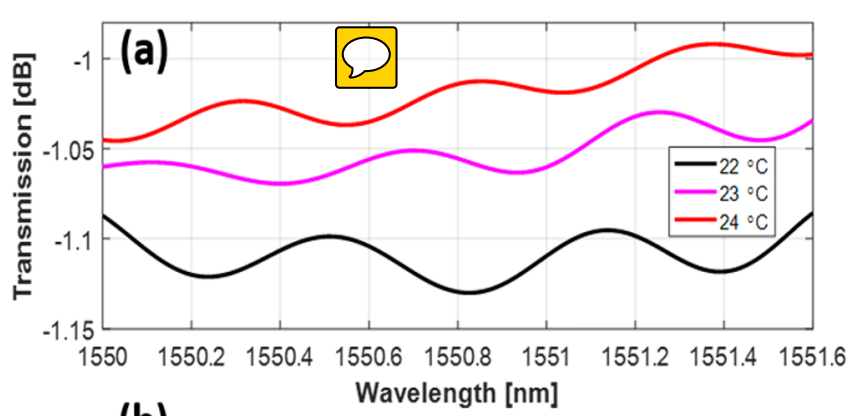

(b)

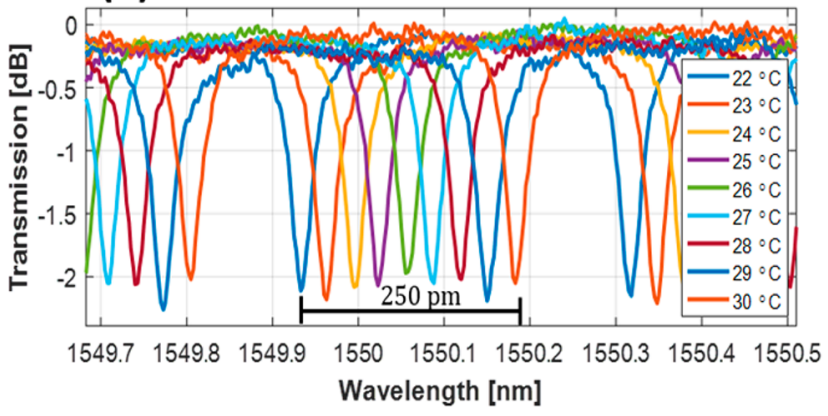

Figure 3. Spectral response of (a) a $\mathrm{SiOC}$-coated $\mathrm{Ge}: \mathrm{SiO}_{2}$ straight waveguide for three different temperatures; (b) a SiOC-coated SiON ring resonator for nine different temperatures.

a SiOC-coated $\mathrm{Ge}: \mathrm{SiO}_{2}$ straight waveguide around $\lambda=1550212$ $\mathrm{nm}$. The Fabry-Pérot fringes, which are less pronounced than 213 in Figure 1(c) because of the lower effective index of the 214 waveguide, reveal $n_{\mathrm{g}}=1.93$. When the temperature is increased 215 by $\mathrm{d} T=1{ }^{\circ} \mathrm{C}$, a wavelength shift of the waveguide transmission 216 spectrum $\mathrm{d} \lambda / \mathrm{d} T=130 \mathrm{pm} /{ }^{\circ} \mathrm{C}$ is observed. According to eq 1,217 this shift corresponds to an effective thermo-optic coefficient 218 $K_{\text {eff }}=1.62 \times 10^{-4}{ }^{\circ} \mathrm{C}^{-1}$, which is more than 1 order of 219 magnitude higher than that of $\mathrm{SiO}_{2}$ waveguides. 220

The enhancement of the waveguide effective TOC is 221 confirmed by results achieved on SiOC-coated SiON wave- 222 guides. Figure $3 \mathrm{~b}$ shows the thermally induced wavelength shift 223 of the transmission spectrum of a microring resonator around a 224 wavelength of $1550 \mathrm{~nm}$. When the temperature increases from 225 $22{ }^{\circ} \mathrm{C}$ to $30{ }^{\circ} \mathrm{C}$, the resonance of the microring is shifted by 226 $250 \mathrm{pm}$. From eq 1 , the resulting $K_{\text {eff }}$ is $3.2 \times 10^{-5}{ }^{\circ} \mathrm{C}^{-1} .227$ Remarkably, a thin layer of SiOC of only $60 \mathrm{~nm}$ thickness and 228 
229 with a confinement factor $\Gamma_{\text {SiOC }}$ of less than $5 \%$ increases by 3 230 times the $K_{\text {eff }}$ of the ring original $\mathrm{SiON}$ waveguides $\left(K_{\mathrm{SiON}}=\right.$ $\left.2311.1 \times 10^{-5}{ }^{\circ} \mathrm{C}^{-1}\right)$. These results confirm that SiOC seems an 232 enabling platform to develop highly efficient reconfigurable 233 photonic systems.

234 The origin of the large TOC of SiOC is related to the 235 volume change in polarizability and, in a minor part, on the 236 thermal expansion. This is the typical situation for high melting 237 point, high hardness, and high elastic moduli materials. Also, 238 near the transparency edge, the polarizability (and $\mathrm{d} n / \mathrm{d} T$ ) 239 rises. ${ }^{17}$ Figure 4 reports the TOC of various materials plotted

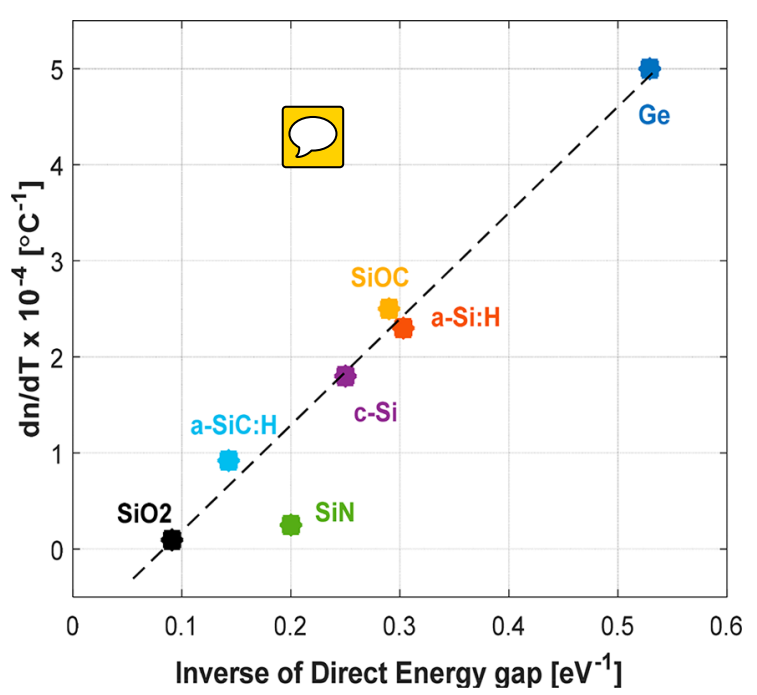

Figure 4. Thermo-optic coefficient dependence on the inverse of the direct band gap energy.

240 against their direct band gap energy $E_{\mathrm{g}}$ at telecom wavelengths. 241 Reported data are obtained from the literature. ${ }^{18,19}$ The 242 thermo-optic coefficient is nearly dependent on the inverse of $243 E_{\mathrm{g}}$ and increases approaching the band gap. Silica is transparent 244 until the deep UV and has a very small TOC, while germanium 245 has the highest coefficient but it is not transparent at telecom 246 wavelengths. The direct band gap of the SiOC film has been 247 determined by using absorption spectra measured with 248 spectroscopic ellipsometry and applying the Tauc relation $249(\alpha h \omega)^{2}=h \omega-E_{\mathrm{g}}$, where $\alpha$ is the absorption coefficient and $250 h \omega$ is the photon energy. ${ }^{20}$ The direct band gap of the SiOC 251 film with $n=2.2$ is calculated as $E_{\mathrm{g}}=3.5 \mathrm{eV}$, which is lower 252 than that of $\mathrm{Si}$ and is responsible for its higher thermo-optic 253 coefficient. As can be seen in Figure 4, SiOC is aligned with the 254 trend of increasing TOC value with decreasing energy band 255 gap.

256 In conclusion, we investigated the thermo-optic effect in 257 optical waveguides integrating high refractive index $(n=2.2)$ $258 \mathrm{SiOC}$ films. Experiments on three different waveguiding 259 structures (channel $\mathrm{SiOC}$ waveguide, $\mathrm{SiOC}$-coated $\mathrm{SiO}_{2}$ 260 waveguide, and $\mathrm{SiOC}$-coated $\mathrm{SiON}$ waveguide) show that 261 the thermo-optic coefficient of SiOC, amounting to $2.5 \times 10^{-4}$ $262{ }^{\circ} \mathrm{C}^{-1}$, is 1 order of magnitude larger than that of dielectric 263 materials conventionally employed in integrated optics and 264 almost twice that of silicon. The high TOC of SiOC was also 265 exploited to achieve a significant enhancement of the TOC of 266 conventional glass waveguides $\left(10 \times\right.$ in $\mathrm{SiO}_{2}, 3 \times$ in $\mathrm{SiON}$ 267 waveguides) by coating the waveguide core with a thin film of $268 \mathrm{SiOC}$. These results demonstrate the potential of $\mathrm{SiOC}$ for the realization of highly efficient thermally tunable photonic 269 integrated platforms.

\section{AUTHOR INFORMATION}

Corresponding Author

*E-mail: faisalahmed.memon@polimi.it.

ORCID

Faisal Ahmed Memon: 0000-0002-4198-3694

Francesco Morichetti: 0000-0002-5858-2811

\section{Author Contributions}

277

F.A.M. fabricated the waveguides and performed experiments 278 for the characterization of the thermo-optic coefficient of 279 SiOC. Results were discussed by all authors. The manuscript 280 was written and approved by all authors.

Notes

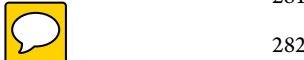

The authors declare no competing financial interest.

\section{ACKNOWLEDGMENTS}

284

This work was partially funded by Fondazione Cariplo Project 285 "Advanced Control Technologies for Integrated Optics 286 (ACTIO)”, Rif. 2016-0881. F.A.M. acknowledges Erasmus 287 Mundus LEADERS Project for the Ph.D. scholarship. The 288 work was mainly performed at Polifab (www.polifab.polimi.it), 289 the micro/nano fabrication facility at Politecnico di Milano, 290 Italy.

\section{REFERENCES}

292

(1) de Ridder, R. M.; Worhoff, K.; Driessen, A.; Lambeck, P. V.; 293 Albers, H. Silicon oxynitride planar waveguiding structures for 294 application in optical communication. IEEE J. Sel. Top. Quantum 295 Electron. 1998, 4, 930.

(2) Muñoz, P.; Micó, G.; Bru, L. A.; Pastor, D.; Pérez, D.; 297 Doménech, J. D.; Fernández, J.; Baños, R.; Gargallo, B.; Alemany, R.; 298 Sánchez, A. M.; Cirera, J. M.; Mas, R.; Domínguez, C. Silicon Nitride 299 Photonic Integration Platforms for Visible, Near-Infrared and Mid- 300 Infrared Applications. Sensors 2017, 17, 2088.

(3) Lim, K. P.; Krishnamurthy, V.; Ying, J. F.; Pu, J.; Wang, Q. 302 Ultrahigh index and low-loss silicon rich nitride thin film for NIR 303 HAMR optics. IEEE Trans. Magn. 2017, 53 (5), 1-7.

(4) Bovington, J.; Wu, R.; Cheng, K.-T.; Bowers, J. E. Thermal stress 305 implications in athermal $\mathrm{TiO}_{2}$ waveguides on a silicon substrate. Opt. 306 Express 2014, 22, 661-666.

(5) Mandracci, P.; Frascella, F.; Rizzo, R.; Virga, A.; Rivolo, P.; 308 Descrovi, E.; Giorgis, F. Optical and structural properties of 309 amorphous silicon-nitrides and silicon-oxycarbides: Application of 310 multilayer structures for the coupling of Bloch Surface Waves. J. Non- 311 Cryst. Solids 2016, 453, 113-117.

312

(6) David, L.; Bhandavat, R.; Barrera, U.; Singh, G. Silicon 313 oxycarbide glass-graphene composite paper electrode for long-cycle 314 lithium-ion batteries. Nat. Commun. 2016, 7, 10998.

(7) Nikas, V.; Gallis, S.; Huang, M.; Kaloyeros, A. E.; Nguyen, A. P. 316 D.; Stesmans, A.; Afanas'ev, V. V. The origin of white luminescence 317 from silicon oxycarbide thin films. Appl. Phys. Lett. 2014, 104, 061906. 318

(8) Memon, F. A.; Morichetti, F.; Melloni, A. Waveguiding light into 319 silicon oxycarbide. Appl. Sci. 2017, 7 (6), 561.

(9) Memon, F. A.; Morichetti, F.; Melloni, A. Integrated photonic 321 devices with silicon oxycarbide. SPIE Photonics Europe Conference 322 2018, 125.

(10) Ryan, J. V.; Pantano, C. G. Synthesis and characterization of 324 inorganic silicon oxycarbide glass thin films by reactive rf-magnetron 325 sputtering. J. Vac. Sci. Technol., A 2007, 25 (1), 153-159.

(11) Miyazaki, H. Structure and optical properties of silicon 327 oxycarbide films deposited by reactive RF magnetron sputtering 328 using a SiC target. Japanese J. of Appl. Physics 2008, 47 (11R), 8287. 329 
330 (12) Du, P.; Wang, X.; Lin, I. K.; Zhang, X. Effects of composition 331 and thermal annealing on the mechanical properties of silicon 332 oxycarbide films. Sens. Actuators, A 2012, 176, 90-98.

333 (13) Tamir, T. Guided-Wave Optoelectronics; Springer, 1988.

334 (14) Robinson, J. T.; Preston, K.; Painter, O.; Lipson, M. First335 principle derivation of gain in high-index-contrast waveguides. Opt. 336 Express 2008, 16, 16659-16669.

337 (15) Melloni, A.; Costa, R.; Monguzzi, P.; Martinelli, M. Ring338 resonator filters in silicon oxynitride technology for dense wavelength339 division multiplexing systems. Opt. Lett. 2003, 28, 1567-1569.

340 (16) http://www.enablence.com/technologies/.

341 (17) Bass, M. Handbook of Optics: Vol. IV - Optical Properties of 342 Materials, Nonlinear Optics, Quantum Optics; McGraw-Hill, 2010.

343 (18) Palik, E. D. Handbook of Thermo-optic Coefficients of Optical 344 Materials with Applications; Academic, 1998.

345 (19) Della Corte, F. G.; Montefusco, M. E.; Moretti, L.; Rendina, I. 346 Study of the thermo-optic effect in hydrogenated amorphous silicon 347 and hydrogenated amorphous silicon carbide between 300 and $500 \mathrm{~K}$ 348 at $1.55 \mu \mathrm{m}$. Appl. Phys. Lett. 2001, 79, 168.

349 (20) Tauc, J. Optical properties and electronic structure of 350 amorphous Ge and Si. Mater. Res. Bull. 1968, 3, 37-46. 